EOMmun Communication et organisation

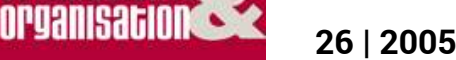

La communication des nouvelles éthiques de

l'entreprise

\title{
Comment communiquer à des parties prenantes aux intérêts contradictoires?
}

Annick Schott-Séraudie

\section{OpenEdition}

\section{Journals}

Édition électronique

URL : http://journals.openedition.org/communicationorganisation/3297

DOI : 10.4000/communicationorganisation.3297

ISSN : 1775-3546

Éditeur

Presses universitaires de Bordeaux

Édition imprimée

Date de publication : 2 janvier 2005

Pagination : 235-245

ISSN : $1168-5549$

Référence électronique

Annick Schott-Séraudie, "Comment communiquer à des parties prenantes aux intérêts

contradictoires? », Communication et organisation [En ligne], 26 | 2005, mis en ligne le 19 juin 2012.

consulté le 30 avril 2019. URL : http://journals.openedition.org/communicationorganisation/3297 ;

DOI : 10.4000/communicationorganisation.3297

Ce document a été généré automatiquement le 30 avril 2019.

(c) Presses universitaires de Bordeaux 


\title{
Comment communiquer à des parties prenantes aux intérêts contradictoires?
}

\author{
Annick Schott-Séraudie
}

1 Les résultats d'une enquête que j'ai menée en 2002 sur la problématique de la culture d'entreprise comme invariant de la connaissance et de l'engagement durable auprès de cinq PME locales non cotées (de 20 à 80 salariés) m'ont conduit aux deux enseignements suivants :

2 Ce qui semble compter pour ces entreprises dans leurs discours et leur pratique peut se


impératif d'éviter d'être agi par des contraintes externes et / ou internes qui pourraient pousser l'action au détriment de l'humain, mais plutôt de tenter d'agir sur ces contraintes quitte à repositionner son action afin de maintenir l'homme au centre de l'entreprise.

3 Les conditions d'existence de cette boucle passent par la co-construction de l'entité «entreprise». Pour ce faire, il est impératif de situer l'ensemble des relations (transversales, ascendantes, descendantes) dans une dynamique de coopération et d'interaction. L'outil importe peu, canal oral, support papier ou multimédia, il est là pour répondre à cette finalité et non pour l'instrumentaliser. Cela signifie que la communauté entreprise pour durer à tout intérêt à faire le pont entre l'Individuel et le Collectif. Ce pont passe par l'acte d'établir ensemble l'échelle du tolérable et celle de l'intolérable. L'entreprise devient alors un tiers symbolique : ni salarié, ni chef d'entreprise, mais des savoir, savoir-faire, savoir-être coopératifs au service de ce tiers « entreprise ».

4 La culture d'entreprise devient alors pour le dirigeant une façon de communiquer par rapport au sens de l'évolution qu'il donne à ce personnage entreprise. Sa façon de communiquer va aussi dépendre de la phase où il situe l'évolution de son entreprise. Cependant s'il essaie de voir ce qui EST (le récit des faits et des actes) il constate que sa sphère de liberté de penser est quelque peu soumise à la sphère du poids économique.

5 Qu'en est-il donc pour une entreprise coté, même au second marché ?

6 Que retiennent ses prescripteurs de ce type de pratique loyale? 
7 La nécessité de se conformer aux normes (lois), la contribution de l'entreprise à la société, voire son obligation de communiquer, renforcent l'empreinte du dirigeant dans la ou les perceptions par les parties prenantes (voir leurs attentes contradictoires cf. ANNEXE 1 et 2) del'entrepriseet deson l'image.

8 D'aucun va rechercher dans la manière dont le dirigeant a de diriger sa LOYAUTE / sa RESPONSABILITE pour confirmer la FIABILITE / la QUALITE des biens et services produits par l'entreprise.

9 L'entreprise (son dirigeant) peut être scrutée à travers sa responsabilité économique, sa responsabilité légale, sa responsabilité éthique, sa responsabilité philanthropique ou volontaire.

10 La responsabilité ECONOMIQUE : à la base l'entreprise doit produire des biens et des services pour faire un profit tout en répondant aux attentes de la société (des consommateurs, clients, usagers, concitoyens). L'objectif : être rentable.

11 La responsabilité LEGALE : l'entreprise doit se conformer aux lois et à la réglementation. L'objectif : obéir à la législation (tout en étant économiquement viable).

12 (Voir loi NRE - politique sociale et politique environnementale cf. Annexe 3)

13 La responsabilité ETHIQUE : les conduites, façons d'agir de l'entreprise sont conformes aux attentes, préférences, valeurs de ce que la société juge comme juste, estimable notamment vis-à-vis de ses parties prenantes. L'objectif : être éthique (tout en obéissant à la législation et en étant économiquement viable)

14 La responsabilité PHILANTROPIQUE ou VOLONTAIRE: l'ensemble des actions que l'entreprise développe poursuit le but d'être une entreprise citoyenne. L'objectif : être une entreprise citoyenne (tout étant éthique, obéissant à la législation et économiquement viable).

Qu'en retiennent certains prescripteurs? Deux enquêtes récentes permettent d'apporter quelques éléments de réponses. L'une concerne les analystes financiers, l'autre les pratiques de gestion des ressources humaines sur la performance économique.

Que retiennent les analystes financiers des informations non financières diffusées par une entreprise cotée?

«Ce qui est gérable est mesurable, ce qui est géré est mesuré »

Une enquête réalisée en 2004 par Judith Saghroun (maître de conférences à Paris X) et Jean-Yves Eglem (professeur à ESCP-EAP) auprès de 25 analystes financiers montre que leur attention s'oriente principalement vers les informations économiques, les autres types d'informations apparaissant comme peu pertinentes.

La performance de l'entreprise est jugée à partir d'informations économiques et financières sont peu prises en compte des informations extra-financières.

«Est performante l'entreprise qui garantit un bon retour sur capitaux investis,

réalise ce qu'elle a promis, respecte ses engagements, a une bonne capacité à

dégager de la trésorerie (des liquidités) et n'est pas trop endettée ».

De plus, les informations extra-financières sont analysées en termes de rentabilité.

Ainsi l'image de l'entreprise perçue, personnalisée par l'image du dirigeant, sa bonne réputation, la qualité de son management est jugée très importante: "malgré les pollutions Total a une superbe image auprès des analystes grâce à l'image et la qualité de management des dirigeants ». 
21 Cette lecture des analystes financiers semblerait signifier que serait autant prise en compte l'image externe (du dirigeant) auprès de ses clients, partenaires, marchés financiers et de la société civile que son image interne auprès de ses salariés. rémunérations, de formation, de conditions de travail, de climat social, de structure de l'emploi ; ceux significatifs de la performance économique concernent la valeur ajoutée, les flux de trésorerie, le résultat résiduel. rémunérations prises en compte se déclinent en écart de rémunération moyenne ouvriers/cadres, rémunération moyenne cadres, 10 salaires supérieurs; celles relatives à la formation concernent le pourcentage de dépense de formation, le pourcentage de stagiaires cadres, les heures de stages payées par personne; les variables en terme de 
conditions de travail retiennent le taux de gravité des accidents du travail, les œuvres sociales, la durée du travail, celles du climat social s'attachent au taux d'absentéisme, au taux total de démission, au taux de démission des cadres, au taux de démission des noncadres, à l'écart de rémunération hommes/femmes cadres; les variables de la structure de l'emploi s'intéressent au pourcentage de cadres, au taux d'embauche, au taux de licenciement.

Côté performance économique, la valeur ajoutée s'intéresse à la richesse distribuée aux différents partenaires, les flux de trésorerie affinent la notion de fonds de roulement, le résultat résiduel est représenté par le résultat net moins le capital investi.

\section{Hypothèses retenues :} des aberrations conduisent au résultat suivant: l'H 1 et l'H 2 sont vérifiées : plus les politiques de rémunération et de formation sont favorables aux salariés, plus la performance économique de l'entreprise est élevée. En effet, l'association entre ces éléments de politique sociale et les éléments de performance économique reste positive et pertinente même à court terme.

- Hypothèse 1 : plus la politique de rémunération est favorable aux salariés, plus la performance économique de l'entreprise est élevée.

- Hypothèse 2 : plus la politique de formation est favorable aux salariés, plus la performance économique de l'entreprise est élevée.

- Hypothèse 3 : plus les conditions de travail sont favorables aux salariés, plus la performance économique de l'entreprise est élevée.

- Hypothèse 4 : plus le climat est défavorable aux salariés, moins la performance économique de l'entreprise est élevée.

- Hypothèse 5 : plus les variables de la structure d'emploi considérées sont au beau fixe, plus la performance économique de l'entreprise est élevée.

Contraintes méthodologiques et résultats.

Si les autres hypothèses n'ont pas été vérifiées, c'est en raison de la logique court termiste induite par le résultat annuel de l'entreprise. La mesure des conditions de travail, du climat social, et des indicateurs de structure de l'emploi par rapport à la performance économique ne peut trouver de résonance et de signification que décalée dans le temps (corrélations moyen long terme).

Les investissements en rémunération et formation sont-ils les seuls capables de mettre en exergue le potentiel créatif de certains salariés pour améliorer les produits et services et assurer la pérennité de l'entreprise ? L'homme au travail est-il un porte-monnaie?

Un salarié heureux, épanoui est-il un salarié performant? Peut-être : à la condition non négligeable que tout son affect ne soit pas sollicité, convoqué, sinon comme l'indique Eric Albert (psychiatre - 2001), il se fragilise puisqu'il projette ce qu'il fait sur ce qu'il est.

Optimiser les ressources humaines, par nécessité économique ; capitaliser sur l'humain : OUI ! À condition que cet investissement sur le potentiel, les talents cachés, ne soit pas assimilé à un investissement sur une chose, un moyen matériel qui ne peut être maximalisé que s'il est détruit pour être changé, renouvelé, et ce en permanence; ou 
mieux que cet investissement ne représente pas une variable d'ajustement... comptable et financière. Comme le précise Hervé Serieyx, « la ressource humaine qui serait stratégique apparaît comme un pur concept à mille lieues du réel ou pire comme un understatement rappelant le rôle de variable d'ajustement que représente le personnel ». Un constat qui a malheureusement tendance se répandre.

Par ailleurs, si nous sommes essentiellement des êtres de communication, qui transformons ce qui agit sur nous, qu'en est-il de la symétrie de l'échange. Celui-ci n'est-il pas orienté pour satisfaire plutôt une partie prenante contre une autre.

Ces deux enquêtes, et d'autres relatives aux analyses de contenu de journaux internes, média sensé régénérer le consensus des salariés ${ }^{1}$, nous indiquent que ce qui est en fait valorisé ce sont les performances économiques de l'activité de l'entreprise et non les savoir-faire des femmes et des hommes qui les impulsent.

\section{BIBLIOGRAPHIE}

Albert E. (2001) N'obéissez plus, Paris, Editions d'organisation.

Beaudoin, JP. (2001) Etre à l'écoute du risque d'opinion, Paris, Editions d'organisation.

De Gaulejac V. (2001), «L'abstraction et la psychologisation du pouvoir », Les cahiers du

changement, $\mathrm{n}^{\circ} 6$, juin 2001, pp. 25-32.

Le Goff J-P. (2000), Les illusions du management, Paris, La Découverte.

Mintzberg H., Ahlstrand B. et Lampel J. (2000), Safari en pays Stratégie, Paris, Ed. Village Mondial, $423 \mathrm{p}$.

Sérieyx H. (2003), Boussole pour temps de brume, Paris, Ed. Village Mondial, p ; 74.

Thévenet M. (2003), Le management, une affaire de proximité, Paris, Editions d'organisation, 273 p.

\section{ANNEXES}

\section{Annexe 1. Ligne comptable et leur cible}

\begin{tabular}{|l|l|}
\hline Ligne comptable & Cible \\
\hline Chiffre d'affaires & Les clients \\
\hline Achats & Les fournisseurs \\
\hline Taxes & Les collectivités locales et territoriales \\
\hline Salaires & Les salariés \\
\hline
\end{tabular}




\begin{tabular}{|l|l|}
\hline Impôts et charges sociales & L'Etat \\
\hline Dividendes & Les actionnaires \\
\hline
\end{tabular}

Annexe 2. Les différentes représentations du monde de l'entreprise par quelques parties prenantes

\begin{tabular}{|l|l|l|l|l|}
\hline & CONCEPT & EVALUATION & STATUT & RISQUE \\
\hline Salarié & Emploi & Niveau de Revenu & professionnel & $\begin{array}{l}\text { Exclusion } \\
\text { sociale }\end{array}$ \\
\hline Management & $\begin{array}{l}\text { Gouvernement de } \\
\text { personnes }\end{array}$ & $\begin{array}{l}\text { Gestion du capital } \\
\text { humain }\end{array}$ & $\begin{array}{l}\text { Positionnement } \\
\text { hiérarchique }\end{array}$ & $\begin{array}{l}\text { Conflit du } \\
\text { travail }\end{array}$ \\
\hline Finances & $\begin{array}{l}\text { Instrument de } \\
\text { production }\end{array}$ & $\begin{array}{l}\text { Ratio Coût } \\
\text { Capital/Métiers }\end{array}$ & $\begin{array}{l}\text { Coûts ou valeur } \\
\text { ajoutée }\end{array}$ & $\begin{array}{l}\text { Rigidité } \\
\text { structurelle" }\end{array}$ \\
\hline Société & $\begin{array}{l}\text { Droit du travail, } \\
\text { droit des affaires }\end{array}$ & Richesse & Insertion sociale & $\begin{array}{l}\text { Destruction } \\
\text { d'emploi }\end{array}$ \\
\hline
\end{tabular}

Inspiré de JP Beaudoin, Etre à l'écoute du risque d'opinion, Paris, Edition d'organisation, 2001, p. 123

\section{Annexe 3. La Loi NRE}

\section{ARTICLE L. 225-102-1 DE LA LOI NRE ET PRINCIPALES DISPOSITIONS DU DECRET D'APPLICATION}

En France, les sociétés sont tenues, depuis la loi NRE (article L. 225-102-1 de la loi n - 2001-420 du 15 mai 2001 relative aux Nouvelles Régulations Economiques), de préciser dans leur rapport de gestion annuel "la manière dont (elles) prennent en compte les conséquences sociales et environnementales de leur activité".

Ces dispositions, qui ne s'appliquent qu'aux sociétés dont les titres sont admis aux négociations sur un marché réglementé, et prennent effet à compter de la publication du rapport annuel portant sur l'exercice ouvert à compter du $1^{\mathrm{er}}$ janvier 2002, ont été précisées dans le décret d'application spécifique (décret $n^{\circ} 2002-221$ du 20 février 2002 pris pour l'application de l'article L. 225-102-1 du code du Commerce et modifiant le décret n 67-236 du 23 mars 1967 sur les sociétés commerciales, publié le 21 février 2002).

\section{Politique sociale}

Effectif total et embauche

Informations relatives aux plans de réduction des effectifs et de sauvegarde de l'emploi ; efforts de reclassement, réembauches

Organisation du temps de travail, durée du travail, absentéisme 
Rémunérations et évolution, charges sociales, égalité professionnelles entre les hommes et les femmes

Relations professionnelles et bilan des accords collectifs

Conditions d'hygiène et sécurité

Formation

Emploi et insertion des travailleurs handicapés

Oeuvres sociales

Importance de la sous-traitance

Prise en compte de l'impact territorial de ses activités en matière d'emploi et de développement régional

Description des relations entretenue avec les associations de réinsertion, de consommation, de défense de l'environnement

Importance de la sous-traitance et de la façon dont la société promeut auprès de ses soustraitants et la manière dont elle s'assure du respect par ses filiales du respect des conventions de l'OIT (Organisation Internationale du Travail)

Prise en compte par les filiales étrangères de l'impact de leurs activités sur le développement régional et les populations locales

\section{Politique environnementale}

Consommation de ressources en eau, matières premières, énergie et mesures prises pour recourir aux énergies nouvelles, pour maîtriser les rejets dans l'eau et dans l'air, atténuer les nuisances olfactives et sonores à partir d'une liste qui sera établie par arrêtés des ministres de l'environnement et de l'industrie

Mesures prises pour limiter les atteintes aux équilibres biologiques, aux milieux naturels, aux espèces animales et végétales

Démarches d'évaluation ou de certification en matière d'environnement

Mesures prises pour assurer la conformité aux dispositions législatives et réglementaires applicables en matière environnementale

Dépenses engagées pour prévenir les conséquences de l'activité de la société sur l'environnement

Existence de services internes de gestion et formation à l'environnement et organisation mise en place pour faire face aux accidents de pollution

Montant des provisions et garanties pour risques en matière environnementale 
Indemnités versées au cours de l'exercice en exécution d'une décision judiciaire en matière environnementale sauf si cette information est de nature à causer un préjudice sérieux à la société dans un litige en cours

Tous les éléments sur les objectifs que la société assigne à ses filiales à l'étranger.

\section{NOTES}

1. D'après les discours de ceux qui sont en charge d'un Journal Interne, celui-ci sert autant à renforcer la cohésion sociale des équipes de travail (satisfaire le besoin de connaître ce qui se passe dans l'entreprise, faire partager les valeurs propres de l'entreprise, combattre la désinformation) qu'à renforcer l'efficacité des équipes de travail (faciliter le travail de chacun par la connaissance des tenants et aboutissants de son activité, expliquer les objectifs, expliquer les nécessités du changement (équipement, organisation) sensibiliser et/ou donner des conseils sur ses questions de santé et sécurité, de droits sociaux, d'économie ou de gestion de l'entreprise.

\section{RÉSUMÉS}

Cet article propose deux études qui montrent que pour des firmes, malgré la diffusion d'informations sociales, voire sociétale, la priorité éthique se concentre toujours davantage sur une partie prenante spécifique (l'actionnaire).

The paper proposes to understand how and to which extent companies are likely to process or no social information

\section{AUTEUR}

\section{ANNICK SCHOTT-SÉRAUDIE}

Annick Schott-Séraudie est Maître de Conférences à l'IUT à l'Université Michel de Montaigne-Bordeaux 3. Elle est membre du GREM/CEMIC. La problématique générale de ses recherches s'articule autour de la question suivante : comment l'entreprise peut-elle traiter tout à la fois la prise individuelle d'autonomie des salariés (équipes - services lignes hiérarchiques) et le renforcement des maillages des actions collaboratives administrés ou structurés par des mises en situation/outils (TIC, dialogue social, dynamisation des activités,..) pour répondre aux facteurs jugés essentiels à son évolution, comme la satisfaction contrastée de différentes parties prenantes, tout en maintenant auprès de chacune de ses parties prenantes un niveau acceptable de « pratique loyale »? Mail : annick.schott@iut.u-bordeaux3.fr 